\title{
Outage Probability for Cooperative Nano Communication in the THz Gap Frequency Range
}

\author{
A. Chaminda J. Samarasekera ${ }^{1}$ and Hyundong Shin ${ }^{2}$ \\ ${ }^{1}$ Kyung Hee University \\ Youngin 446-701 - Korea \\ [e-mail: samarasekera@khu.ac.kr] \\ ${ }^{2}$ Kyung Hee University \\ Youngin 446-701 - Korea \\ [e-mail: hshin@khu.ac.kr] \\ *Corresponding author: Hyundong Shin
}

Received July 27, 2016; revised October 10, 2016; revised November 30, 2016; accepted December 9, 2016;

published January 31, 2017

\begin{abstract}
Nanotechnology has provided a set of tools that the engineers can use to design and manufacture integrated nano devices, which are capable of performing sensing, computing, data storing and actuation. One of the main hurdles for nano devices has been the amount of power that it can generate for transmission of data. In this paper, we proposed cooperative nano communication in the Terahertz $(\mathrm{THz}) \mathrm{Gap}$ frequency band to increase the range of transmission. Outage probability (OP) performances for the proposed cooperative nano communication networks in the $\mathrm{THz}$ band $(0.1-10 \mathrm{THz})$ have been evaluated for the following scenarios; A) A single decode-and-forward (DF) relay over independent identically distributed (i.i.d.) Rayleigh fading channels, B) DF multi-relay network with best relay selection (BRS) over i.i.d. Rayleigh fading channels, and C) DF multi-relay network with multiple hops with BRS over i.i.d. Rayleigh fading channels. The results show that the transmission distance can be improved significantly by employing DF relays. Also, it is shown that by increasing the number of hops in a relay the OP performance is marginally degraded. The analytical results have been verified by Monte-Carlo simulation.
\end{abstract}

Keywords: Terahertz Band, Nanonetworks, Channel Model, Cooperative Communication, Outage Probability, Graphene. 


\section{Introduction}

The advancement of nanotechnology in the past decade has made it possible for many new and innovative applications. Nanotechnology has enabled devices to be built in the range of 1 to a few hundred nanometers, which are capable of performing basic tasks as sensing, relaying, etc [1], [2].

Richard Feynman first envisioned nanotechnology in 1959, in a speech titled "There's Plenty of Room at the Bottom", which has enabled engineers to design and manufacture integrated nano-devices for many basic applications. To perform more complex functions there is a need for communication, control and coordination among the nano machines [3]. Nanonetworks will enable advancements in the fields of biomedical, environmental, military, first responders', etc. Control methods for nanomechatronic systems with respect to signaling, structure, and functions are presented in [4]. Bionanosensor networks represent a new type of wireless sensor network, developed by the combined implementation of nanotechnology and materials science theory [5]. A bionanosensor network is composed of a number of nanomachines with the ability to sense chemical signals. A conceptual neuronal nanomachine acting at cellular level, with the main aim of employing nano devices to assist or restore not working neurons is presented in [6]. Nano sensors are actually nano machines that are capable of taking advantage of properties of novel nanometerials. Graphene is such a nanomaterial, that can detect chemical compounds as low as one part per billion, presence of viruses and bacteria [7].

Graphene is a single layer of carbon packed in a hexagonal lattice, with a carbon-carbon distance of $0.142 \mathrm{~nm}$. It is the first truly two-dimensional crystalline material and it is representative of a whole class of 2D materials, including for example single layer of BoronNitride(BN) and Molybdenum-disulphide $\mathrm{MoS}_{2}$, both produced after 2004 [8]. Graphene has a number of properties that makes it interesting for several different applications. It is extremely thin, mechanically very strong, flexible, transparent and a conductor. Furthermore, it can be used as a flexible electronic and gas sensor. Due to its low weight, it is possible to have many applications in aircrafts, satellites and others [8]. Graphene Nanoribbons and Carbon Nanotubes have enabled the development of nano-batteries, nano-memories, nanosensors and nano-actuators from unique properties observed in nanomaterials and its derivatives [9]. Graphene based nano antennas in the $\mathrm{THz}$ frequency band have been proposed and evaluated in [10], [11] and [12].

$\mathrm{THz}$ waves are located between millimeter waves and infrared lightwaves in the electromagnetic spectrum, which has been rarely utilized except for astronomy and fields related to astronomy [13]. Feasibility of using the THz waves in the range of $0.1 \mathrm{THz}-10$ $\mathrm{THz}$ for future wireless communications and technologies associated, has been investigated by Klein-Ostmann and Nagatsuma in [14] and by Federici and Moeller in [15]. A review of $\mathrm{THz}$ wave technologies and applications related to communications are discussed in [16] by Ho-Jin Song and Nagatsuma. Ultra-broadband terahertz communication, based on directed non-line-of-sight transmissions have been proposed in [17], which have applications in wireless local area networks (WLANs) and wireless personal area networks (WPANs). The total path loss of a propagating wave in the Terahertz Band depends on the transmission distance and the system frequency, as well as the composition of the transmission medium at a molecular level [7]. A channel model for electromagnetic wireless nanonetworks in the 
terahertz band has been introduced in [7], furthermore the capacity of that nanonetwork has also been analyzed in it.

One of the hurdles for nano machines has been the amount of power that the nano machines are capable of producing, which has been minimal so far. Therefore, it is important that nano networks are created and that information can be communicated through relaying to command centers. By making advances in wireless nanosensor networks, it is possible to enable health monitoring, surveillance of weapons of mass destruction, etc [7].

A Channel-aware forwarding scheme for Nano sensor networks in the terahertz band have been investigated in [18]. Where the channel-aware forwarding scheme makes forwarding decisions considering the nature of the frequency selective peculiarity of the terahertz channels. In [19], the authors have discussed about a cluster-based forwarding scheme for body area network for e-health application using nano-electromagnetic communication. In this paper, we have proposed using cooperative communication techniques to increase the transmission range of nano devices. The proposed cooperative nano communication networks' OP performance has been evaluated. The nano machines transfer the data from end-to-end using decode-and-forward relaying method. When there are multiple relays, best relay selection scheme has been used. The channel model for electromagnetic communication for nano-devices developed in [7] has been used and the THz band (0.1 -10.0 $\mathrm{THz}$ ) has been utilized for communication.

The rest of this paper has been organized as follows. In Section II the propagation and relay models are explained. In Section III, outage probability analysis has been calculated for: A) a single decode-and-forward relay, B) multiple decode-and-forward relays with best relay selection, and C) multiple decode-and-forward relays with multiple decode-and-forward hops. In Section IV, the numerical calculations are validated with simulations and the results are investigated. Finally the paper is summarized and conclusions are presented in Section V.

\section{Propagation and Relaying Models}

\subsection{Terahertz Propagation Model}

In [7], a propagation model for Graphene-based electromagnetic nano-tranceivers in the frequency range of the Terahertz band $(0.1-10.0 \mathrm{THz})$ is given. The path loss and noise power has been calculated as follows in [7]:

\subsubsection{Pathloss}

The total pathloss for an electromagnetic wave in the Terahertz band can be written as

$$
A(f, d)=A_{\text {spread }}(f, d)+A_{\text {absorption }}(f, d), \#(1)
$$

where $A_{\text {spread }}(f, d)$ denotes the spreading loss of the signal and $A_{\text {absorption }}(f, d)$ denotes the signal attenuation due to molecular absorption.

The spreading loss can be written as

$$
A_{\text {spread }}(f, d)=20 \log \left(\frac{4 \pi f d}{c}\right) \text {.\#(2) }
$$

Molecular absorption attenuation can be written as

$$
A_{\text {absorption }}=\frac{1}{\tau(f, d)}=e^{k(f) d} \text {.\#(3) }
$$

Transmittance of medium, $\tau$, has been obtained using the Beer-Lambert Law as 


$$
\tau(f, d)=\frac{P_{0}}{P_{i}}=e^{-k(f) d}, \#(4)
$$

where $f$ is the frequency of the electromagnetic wave considered, $d$ is the total path length, $P_{0}$ and $P_{i}$ are the radiated and incident powers and $k$ is the medium absorption coefficient. The absorption coefficient for the composition of molecules found along the path can be defined as

$$
k(f)=\sum_{i, g} k^{i, g}(f), \#(5)
$$

where absorption coefficient of the isotopologue $i$ of gas $g$, is $k^{i, g}$. Absorption coefficient of the isotopologue $i$ of gas $g, k^{i, g}$, in $m^{-1}$, can be written as

$$
k^{i, g}(f)=\frac{p}{p_{0}} \frac{T_{s t p}}{T} Q^{i, g} \sigma^{i, g}(f), \#(6)
$$

where $Q^{i, g}$ is the volumetric density, in molecules $/ \mathrm{m}^{3}$ at pressure $p$ and temperature T. $p_{0}$ and $T_{s t p}$ are standard pressure and temperature values and $\sigma^{i, g}$ is the absorption cross section for the isotopologue $i$ of gas $g$ in $\mathrm{m}^{2} /$ molecule.

The total number of molecules per unit volume, $Q^{i, g}$, of the isotopologue $i$ of gas $g$ for the given gas mixture can be calculated as follow in molecules $/ \mathrm{m}^{3}$ using the Ideal Gas Law as

$$
Q^{i, g}=\frac{p}{R T} q^{i, g} N_{A}, \#(7)
$$

where $p$ is pressure, $T$ is temperature, and $R$ is the gas constant. $q^{i, g}$ is the mixing ratio for the isotopoloque $i$ of gas $g$, and $N_{A}$ stand for the Avogadro constant.

$\sigma^{i, g}$ is the absorption cross section of the isotopologue $i$ of gas $g$ and can be calculated as follows

$$
\sigma^{i, g}=S^{i, g} G^{i, g}(f), \#(8)
$$

where $S^{i, g}$ is the line intensity and it can be directly obtained from the High Resolution Transmission (HITRAN) database. $S^{i, g}$ defines the strength of the absorption by a specific type of molecules. To obtain the line shape, $G^{i, g}$, the resonant frequency $f_{c}^{i, g}$ for the isotopologue $i$ of gas $g$ has to be determined as follows

$$
f_{c}^{i, g}=f_{c 0}^{i, g}+\delta^{i, g} \frac{p}{p_{0}}
$$

where $f_{c 0}^{i, g}$ is the zero-pressure position of the resonance and $\delta^{i, g}$ is the linear pressure shift. These parameters can be directly obtained from the HITRAN database.

Lorentz half-width, $\alpha_{L}^{i, g}$, the amount of broadening depends on the molecules involved in collisions. Lorentz half-width can be obtained as a function of air-broadened half-width $\alpha_{0}^{\text {air }}$ and self-broadened half-width $\alpha_{0}^{i, g}$ as follows

$$
\alpha_{L}^{i, g}=\left[\left(1-q^{i, g}\right) \alpha_{0}^{i, g}+q^{i, g} \alpha_{0}^{i, g}\right]\left(\frac{p}{p_{0}}\right)\left(\frac{T_{0}}{T}\right)^{\gamma}, \#(10)
$$

where $q^{i, g}$ is the mixing ratio for the isotopologue $i$ of gas $g, p$ is the system pressure, $p_{0}$ is the reference pressure, $T_{0}$ is the reference temperature, $T$ is the system temperature and $\gamma$ is the temperature broadening coefficient. The values for $\gamma, \alpha_{0}^{\text {air }}$ and $\alpha_{0}^{i, g}$ can be directly obtained from the HITRAN database.

Van Vleck-Wisskopf asymmetric line shape [20] has been considered for the representation of molecular absorption, the frequency band considered is relatively low compared to infrared and light bands 


$$
F^{i, g}(f)=100 c \frac{\alpha_{L}^{i, g}}{\pi} \frac{f}{f_{c}^{i, g}}\left[\frac{1}{\left(f-f_{c}^{i, g}\right)^{2}+\left(\alpha_{L}^{i, g}\right)^{2}}+\frac{1}{\left(f+f_{c}^{i, g}\right)^{2}+\left(\alpha_{L}^{i, g}\right)^{2}}\right], \#
$$

where $f$ is the considered frequency in the electromagnetic waves, $c$ is the speed of light in the vacuum, $\alpha_{L}^{i, g}$ is the Lorentz half-width coefficient for the isotopologue $i$ of gas $g$ and $f_{c}^{i, g}$ is the resonant frequency for the isotopologue $i$ of gas $g$.

To account for the continuum absorption at the far ends of the line shape it was first proposed in [21] and subsequently used in [7] as follows

$$
G^{i, g}(f)=\frac{f}{f_{c}^{i, g}} \frac{\tanh \left(\frac{h c f}{2 k_{B} T}\right)}{\tanh \left(\frac{h c f_{c}^{i, g}}{2 k_{B} T}\right)} F^{i, g}(f), \#(12)
$$

where $h$ is the Planck constant, $c$ is the speed of light in the vacuum, $k_{B}$ is the Boltzmann constant and $T$ is the system temperature.

\subsubsection{Noise Power}

The total noise of the system contains molecular absorption noise, antenna noise temperature, system noise temperature,...etc.

$$
T_{\text {noise }}=T_{\text {system }}+T_{\text {molecular }}+T_{\text {other }} \#(13)
$$

The noise power at the receiver can be calculated as follows for a given bandwidth

$$
P_{n}(f, d)=\int_{B} N(f, d) d f=k_{B} \int_{B} T_{\text {noise }}(f, d) d f \text {,\#(14) }
$$

where $f$ is the frequency in the electromagnetic wave considered, $d$ is the transmission distance, $N$ is the noise power spectral density, $k_{B}$ is the Boltzmann constant and $T_{\text {noise }}$ is the equivalent noise temperature.

It is assumed for this paper, that the graphene based electronics devices are very low noise and therefore, only molecular noise absorption is considered. Molecular absorption noise introduced during the propagation of the electromagnetic waves in the terahertz band can be calculated as follows. The parameter that measures this phenomenon is called the emissivity of the channel, $\varepsilon$

$$
\varepsilon(f, d)=1-\tau(f, d), \#(15)
$$

where $f$ is the frequency in the electromagnetic wave that is considered, $d$ is the total path length traveled and $\tau$ is the transmissivity of the medium given from equation (4) [7].

The noise temperature due to molecular absorption $T_{\text {molecular }}$ that an omnidirectional antenna detects from the medium can be obtained as follows in Kelvin

$$
T_{\text {molecular }}(f, d)=T_{0} \varepsilon(f, d) \text {, \#(16) }
$$

where $f$ is the frequency of the electromagnetic wave that is considered, $d$ is the path length traveled, $T_{0}$ is the reference temperature and $\varepsilon$ is the emissivity of the channel [7] .

\subsection{Relaying Models}

In this paper, decode-and-forward relaying scheme has been used at all relays and hops, and where there are multiple relays best relay selection scheme has been employed, over Rayleigh fading channels 


\subsubsection{Single DF Relay}

A cooperative nano relaying network as in Fig. $\mathbf{1}$ is considered. Where the data is transmitted from the source nano machine to the destination nano machine through a relaying nano machine. Where $h_{s r}$ denotes the channel coefficient of the link Source to Relay and $h_{r d}$ denotes the channel coefficient of the link Relay to Destination.

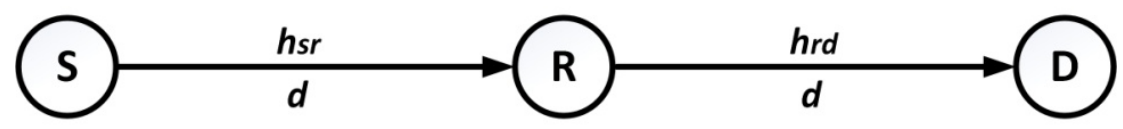

Fig. 1. Single DF Relaying

The instantaneous end-to-end signal-to-noise ratio (SNR) for the cooperative nano relay can be written as

$$
\gamma_{d}=\min \left(\alpha_{s r}\left|h_{s r}\right|^{2}, \alpha_{r d}\left|h_{r d}\right|^{2}\right), \#(17)
$$

where $\alpha_{i}=\frac{s_{i} A_{i}{ }^{-1}}{N_{i}}$, and $S_{i}$ represents transmitted signal power, $A_{i}$ represents total pathloss for electromagnetic wave in the Terahertz band due to molecular absorption attenuation and spreading loss, $N_{i}$ is total noise. $\gamma_{s r}=\alpha_{s r}\left|h_{s r}\right|^{2}$, represents Source to Relay SNR and $\gamma_{r d}=\alpha_{r d}\left|h_{r d}\right|^{2}$, represents Relay to Destination SNR. We have assumed $\beta_{s r}$ and $\beta_{r d}$ as the Rayleigh fading parameters of the channel. Furthermore, $\gamma_{d}$ represents the end-to-end SNR for $S \rightarrow R \rightarrow D$.

\subsubsection{Multiple DF Relays with BRS}

A cooperative nano relaying network as in Fig. $\mathbf{2}$ is considered, where the data is transmitted from the source nano machine to the destination nano machine through relaying nano machines. Where $\boldsymbol{h}_{\boldsymbol{s}}$ denotes the channel coefficient of the link $\boldsymbol{S} \rightarrow \boldsymbol{R}_{\boldsymbol{k}}$ and $\boldsymbol{h}_{\boldsymbol{r}_{\boldsymbol{k}} \boldsymbol{d}}$ denotes the channel coefficient of the link $\boldsymbol{R}_{\boldsymbol{k}} \rightarrow \boldsymbol{D}$.

Therefore, the end-to-end SNR at the destination can be written as follows

$$
\gamma_{d}=\max _{k=1,2, \ldots, K}\left(\gamma_{k}\right), \#(18)
$$

and $\gamma_{k}$ can be determined by

$$
\gamma_{k}=\min _{k=1,2, \ldots, K}\left(\alpha_{s r_{k}}\left|h_{s r_{k}}\right|^{2}, \alpha_{r_{k} d}\left|h_{r_{k} d}\right|^{2}\right) \text {.\#(19) }
$$




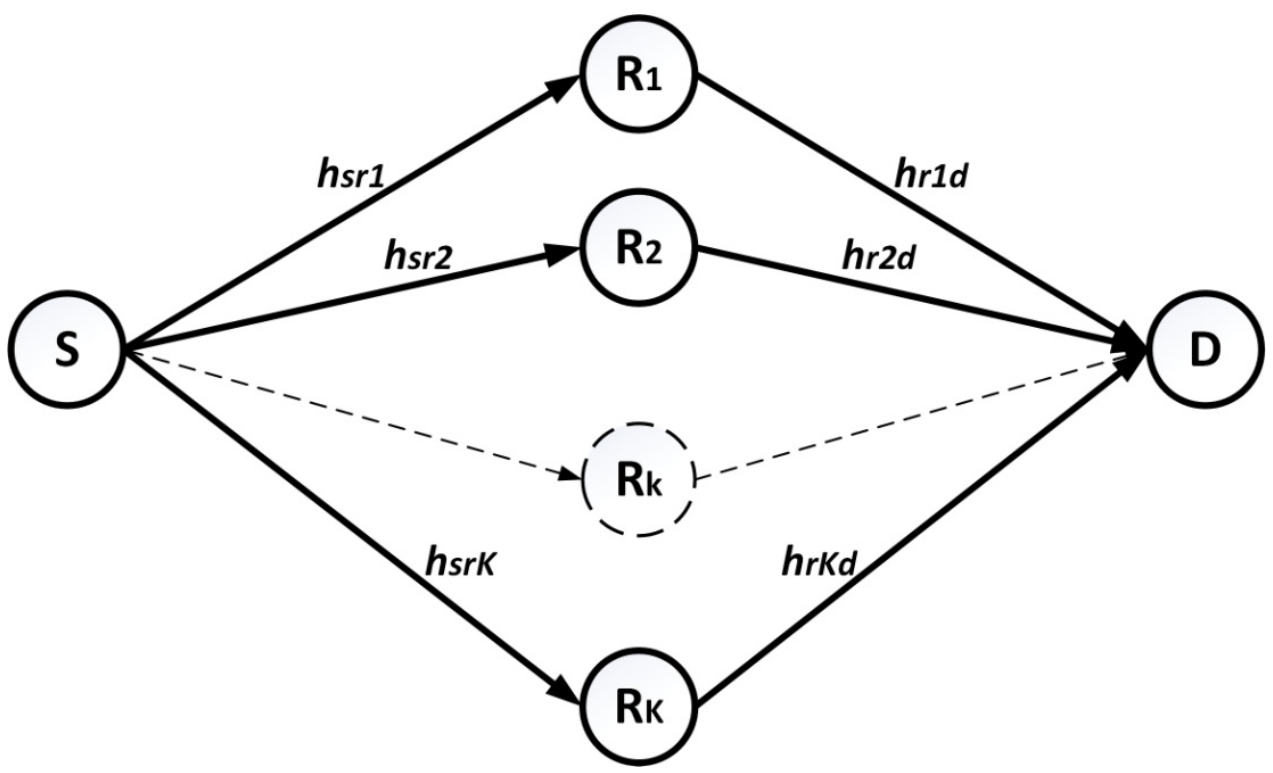

Fig. 2. Multiple DF Relays with BRS

Where $\alpha_{i}=\frac{S_{i} A_{i}^{-1}}{N_{i}}$, and $S_{i}$ represents transmitted signal power, $A_{i}$ represents total pathloss for electromagnetic wave in the Terahertz band due to molecular absorption attenuation and spreading loss, $N_{i}$ is total noise. $\gamma_{s r_{k}}=\alpha_{s r_{k}}\left|h_{s r_{k}}\right|^{2}$, represents $S \rightarrow R_{k}$ SNR and $\gamma_{r_{k} d}=\alpha_{r_{k} d}\left|h_{r_{k} d}\right|^{2}$, represents $R_{k} \rightarrow D$ SNR. $\gamma_{k}$ represents the SNR for the each particular relay. We have assumed $\beta_{s r_{k}}$ and $\beta_{r_{k} d}$ as the Rayleigh fading parameters of the channels. Furthermore, $\gamma_{d}$ represents the end-to-end SNR for $S \rightarrow R_{k} \rightarrow D$.

\subsubsection{Multiple DF Relays with Multiple DF Hops using BRS}

A cooperative nano relaying network as in Fig. 3 is considered, where the data is transmitted from the source nano machine to the destination nano machine through relaying nano machines. Where $h_{s r_{1 k}}$ denotes the channel coefficient of the link $S \rightarrow R_{1 k}$ and $h_{r_{N k} d}$ denotes the channel coefficient of the link $R_{N k} \rightarrow D$. The end-to-end SNR for the above cooperative nano relaying network in Fig. 3 can be written as

and $\gamma_{k}$ can be determined by

$$
\gamma_{d}=\max _{k=1,2, \ldots, K}\left(\gamma_{k}\right), \#(20)
$$

$$
\gamma_{k}=\min _{k=1,2, \ldots, K}\left(\alpha_{s r_{1 k}}\left|h_{s r_{1 k}}\right|^{2}, \ldots, \alpha_{r_{N k} d}\left|h_{r_{N k} d}\right|^{2}\right) . \#(21)
$$




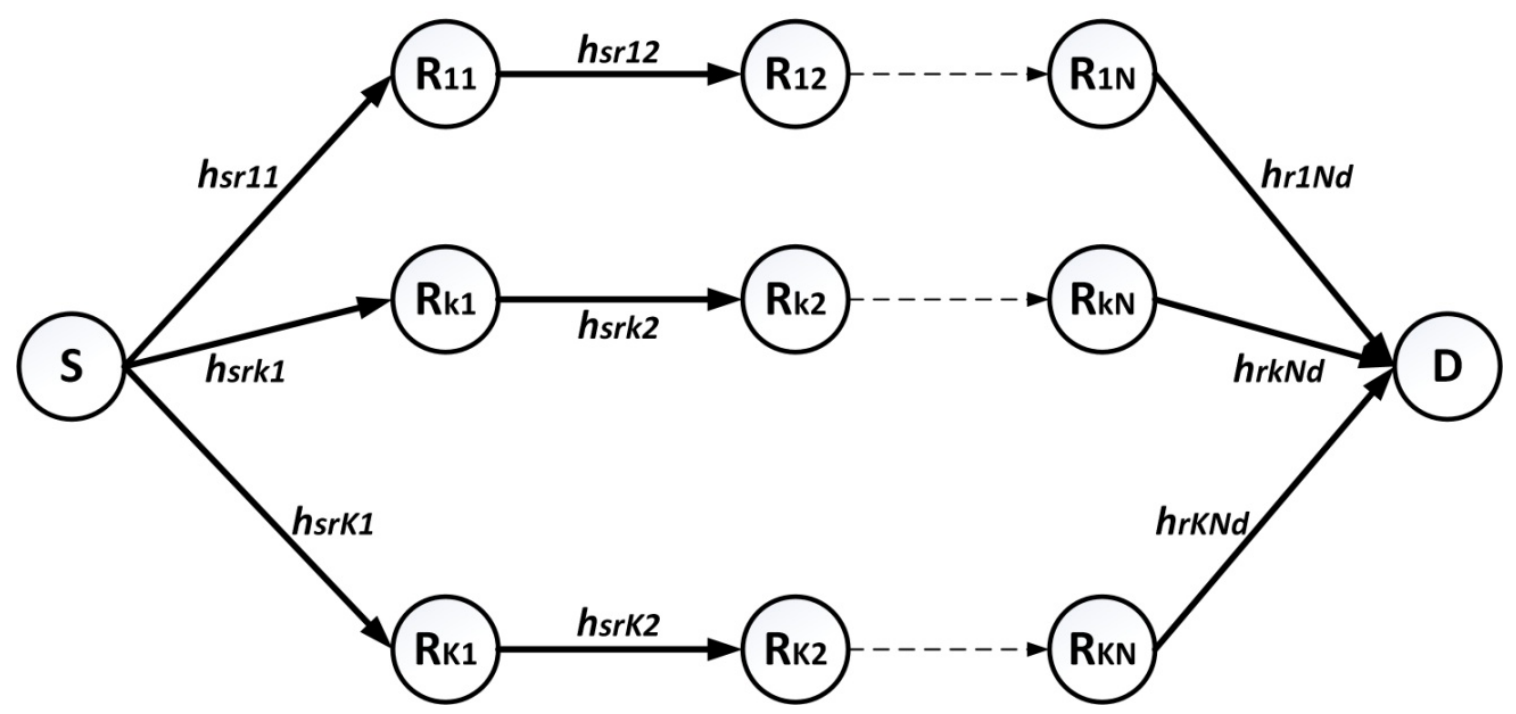

Fig. 3. Multiple DF Relays with Multiple Hops using BRS

Where $\alpha_{i}=\frac{S_{i} A_{i}{ }^{-1}}{N_{i}}$, and $S_{i}$ represents transmitted signal power, $A_{i}$ represents total pathloss for electromagnetic wave in the Terahertz band due to molecular absorption attenuation and spreading loss, $N_{i}$ is total noise. $\gamma_{1 k}=\alpha_{s r_{1 k}}\left|h_{s r_{1 k}}\right|^{2}$, represents $S \rightarrow R_{1 k}$ SNR and $\gamma_{N k}=\alpha_{s r_{N k}}\left|h_{s r_{N k}}\right|^{2}$, represents $R_{N k} \rightarrow D$ SNR. We have assumed $\beta_{1 k}$ and $\beta_{N k}$ as the Rayleigh fading parameters of the channels. Furthermore, $\gamma_{d}$ represents the end-to-end SNR for $S \rightarrow R_{N k} \rightarrow D$.

\section{Outage Probability}

Outage probability is the probability that the instantaneous SNR at the receiver falls below a threshold SNR $\gamma_{t h}$.

\subsection{Single DF Relay}

The OP for a single DF Relay can be calculated as follows. Since $\gamma_{s r}$ and $\gamma_{r d}$ are independent Rayleigh distributed random variables, the cumulative distributive functions (CDFs) for $\gamma_{s r}$ and $\gamma_{r d}$ can be written as

and

$$
F_{\gamma_{s r}}\left(\gamma_{t h}\right)=1-e^{-\left(\frac{\gamma_{t h}^{2}}{2 * \Omega_{s r}^{2}}\right)}
$$

$$
F_{\gamma_{r d}}\left(\gamma_{t h}\right)=1-e^{-\left(\frac{\gamma_{t h}^{2}}{2 * \Omega_{r d}^{2}}\right)}
$$

The end-to-end CDF of $\gamma_{d}$ of the relay can be calculated as

$$
F_{\gamma_{d}}\left(\gamma_{t h}\right)=\left[1-\left(1-F_{\gamma_{s r}}\left(\gamma_{t h}\right)\right)\left(1-F_{\gamma_{r d}}\left(\gamma_{t h}\right)\right)\right] . \#(24)
$$

After substituting (22),(23) in (24), and performing some mathematical manipulations, $F_{\gamma_{d}}\left(\gamma_{t h}\right)$ can be written as

$$
F_{\gamma_{d}}\left(\gamma_{t h}\right)=1-e^{-\left(\frac{\gamma_{t h}^{2}}{2 * \Omega_{s r}^{2}}+\frac{\gamma_{t h}^{2}}{2 * \Omega_{r d}^{2}}\right)}
$$


Therefore, the OP for the single DF relay over i.i.d. Rayleigh fading channels for cooperative nano relaying can be written as follows by substituting the values for $\Omega_{s r}=\alpha_{s r} * \beta_{s r}$ and $\Omega_{r d}=\alpha_{r d} * \beta_{r d}$ in (25).

$$
P_{\text {Out }}(f, d)=1-e^{-\left(\frac{\gamma_{t h}^{2}}{2 *\left(\alpha_{s r} * \beta_{s r}\right)^{2}}+\frac{\gamma_{t h}^{2}}{2 *\left(\alpha_{r d} * \beta_{r d}\right)^{2}}\right)}, \#(26)
$$

where $\alpha_{s r}=\frac{S_{s r}(f, d) * A_{s r}^{-1}(f, d)}{N_{s r}(f, d)}$ and $\alpha_{r d}=\frac{S_{r d}(f, d) * A_{r d}^{-1}(f, d)}{N_{r d}(f, d)} . S_{i}(f, d)$ is the transmitted signal power, $A_{i}(f, d)$ is the total pathloss and $N_{i}(f, d)$ is the noise power. $\beta_{s r}$ and $\beta_{r d}$ are the Rayleigh channel fading parameters.

\subsection{Multiple DF Relays with BRS}

The OP for multiple DF relays with BRS over Rayleigh fading channels can be calculated as follows. The CDF of the end-to-end SNR $F_{\gamma_{d}}\left(\gamma_{t h}\right)$ at the destination with BRS can be expressed as [22], [23]

$$
F_{\gamma_{d}}\left(\gamma_{t h}\right)=\prod_{k=1}^{K} F_{\gamma_{k}}\left(\gamma_{t h}\right), \#(27)
$$

where $F_{\gamma_{k}}\left(\gamma_{t h}\right)$ can be express as

$$
F_{\gamma_{k}}\left(\gamma_{t h}\right)=\left[1-\left(1-F_{\gamma_{s r_{k}}}\left(\gamma_{t h}\right)\right)\left(1-F_{\gamma_{r_{k}}}\left(\gamma_{t h}\right)\right)\right] . \#(28)
$$

The CDFs of $F_{\gamma_{s r_{k}}}\left(\gamma_{t h}\right)$ and $F_{\gamma_{r_{k}}}\left(\gamma_{t h}\right)$ can be written as

and

$$
F_{\gamma_{s r_{k}}}\left(\gamma_{t h}\right)=1-e^{-\left(\frac{\gamma_{t h}^{2}}{2 * \Omega_{s r_{k}}^{2}}\right)}
$$

$$
F_{\gamma_{r_{k} d}}\left(\gamma_{t h}\right)=1-e^{-\left(\frac{\gamma_{t h}^{2}}{2 * \Omega_{r_{k} d}^{2}}\right)}, \#(30)
$$

since $\gamma_{s r_{k}}$ and $\gamma_{r_{k} d}$ are independent Rayleigh distributed random variables.

By substituting (29),(30) in (28) and after performing some mathematical manipulations it is possible to write $F_{\gamma_{k}}\left(\gamma_{t h}\right)$ as

$$
F_{\gamma_{k}}\left(\gamma_{t h}\right)=1-e^{-\left(\frac{\gamma_{t h}^{2}}{2 * \Omega_{s r_{k}}^{2}}+\frac{\gamma_{t h}^{2}}{2 * \Omega_{r_{k} d}^{2}}\right)}, \#(31)
$$

and by substituting (31) in (27), it can be written as follows

$$
F_{\gamma_{d}}\left(\gamma_{t h}\right)=\prod_{k=1}^{K} 1-e^{-\left(\frac{\gamma_{t h}^{2}}{2 * \Omega_{s r_{k}}^{2}}+\frac{\gamma_{t h}^{2}}{2 * \Omega_{r_{k} d}^{2}}\right)} . \#(32)
$$

For i.i.d. Rayleigh fading channels it is possible to further simplify (32), and can be written as

$$
F_{\gamma_{d}}\left(\gamma_{t h}\right)=\left[1-e^{-\left(\frac{\gamma_{t h}^{2}}{2 * \Omega_{s r_{k}}^{2}}+\frac{\gamma_{t h}^{2}}{2 * \Omega_{r_{k} d}^{2}}\right)}\right]^{K}
$$

Applying the following mathematical identity 
to (33), it can be written as

$$
(1+x)^{n}=\sum_{k=0}^{n}\left(\begin{array}{l}
n \\
k
\end{array}\right) x^{k}
$$

$$
F_{\gamma_{d}}\left(\gamma_{t h}\right)=\sum_{j=0}^{K}\left(\begin{array}{c}
K \\
j
\end{array}\right)(-1)^{j} e^{-j\left(\frac{\gamma_{t h}^{2}}{2 * \Omega_{s r_{k}}^{2}}+\frac{\gamma_{t h}^{2}}{2 * \Omega_{r_{k} d}^{2}}\right)}
$$

Therefore, the OP for the multiple DF relays with BRS over i.i.d. Rayleigh fading channels for cooperative nano relaying can be written as follows by substituting the values for $\Omega_{s r_{k}}=\alpha_{s r_{k}} * \beta_{s r_{k}}$ and $\Omega_{r_{k} d}=\alpha_{r_{k} d} * \beta_{r_{k} d}$ in (35) as

$$
P_{\text {Out }}(f, d)=\sum_{j=0}^{K}\left(\begin{array}{c}
K \\
j
\end{array}\right)(-1)^{j} e^{-j\left(\frac{\gamma_{t h}^{2}}{2 *\left(\alpha_{s r_{k}} * \beta_{s r_{k}}\right)^{2}}+\frac{\gamma_{t h}^{2}}{2 *\left(\alpha_{\left.r_{k} d^{*} \beta_{r_{k}}\right)^{2}}\right)}\right.}, \#(36)
$$

where $\alpha_{s r_{k}}=\frac{s_{s r_{k}}(f, d) A_{s r_{k}}^{-1}(f, d)}{N_{s r_{k}}(f, d)}$ and $\alpha_{r_{k} d}=\frac{S_{r_{k} d}(f, d) A_{r_{k} d}^{-1}(f, d)}{N_{r_{k}} d(f, d)} . S_{i}(f, d)$ is the transmitted signal power, $A_{i}(f, d)$ is the total pathloss and $N_{i}(f, d)$ is the noise power. $\beta_{s r_{k}}$ and $\beta_{r_{k} d}$ are the Rayleigh channel fading parameters.

\subsection{Multiple DF Relays with Multiple DF Hops using BRS}

The OP for a multiple DF relays with Multiple DF hops as in Fig. 3 can be calculated as follows. The CDF of the end-to-end SNR $F_{\gamma_{d}}\left(\gamma_{t h}\right)$ at the destination can be expressed as

$$
F_{\gamma_{d}}\left(\gamma_{t h}\right)=\prod_{k=1}^{K} F_{\gamma_{k}}\left(\gamma_{t h}\right)
$$

where $F_{\gamma_{k}}\left(\gamma_{t h}\right)$ can be expressed as

$$
F_{\gamma_{k}}\left(\gamma_{t h}\right)=\left[1-\left(1-F_{\gamma_{s r_{1, k}}}\left(\gamma_{t h}\right)\right) \prod_{n=1}^{N}\left(1-F_{\gamma_{n, k} d}\left(\gamma_{t h}\right)\right)\right] . \#(38)
$$

The CDFs of $F_{s r_{n, k}}\left(\gamma_{t h}\right)$ and $F_{r_{n, k} d}\left(\gamma_{t h}\right)$ can be written as follows

and

$$
F_{\gamma_{s r_{n, k}}}\left(\gamma_{t h}\right)=1-e^{-\left(\frac{\gamma_{t h}^{2}}{2 * \Omega_{s r_{n, k}}^{2}}\right)}
$$

$$
F_{\gamma_{r_{n, k} d}}\left(\gamma_{t h}\right)=1-e^{-\left(\frac{\gamma_{t h}^{2}}{2 * \Omega_{r_{n, k}}^{2}}\right)}, \#(40)
$$

since $\gamma_{s r_{n, k}}$ and $\gamma_{r_{n, k} d}$ are independent Rayleigh distributed varibles.

By substituting (39), (40) in (38), $F_{\gamma_{k}}\left(\gamma_{t h}\right)$ can be written as

$$
F_{\gamma_{k}}\left(\gamma_{t h}\right)=\left[1-e^{-\left(\frac{\gamma_{t h}^{2}}{2 * \Omega_{s r_{n, k}}^{2}}\right)} \prod_{n=1}^{N} e^{-\left(\frac{\gamma_{t h}^{2}}{2 * \Omega_{r_{n, k}}^{2}}\right)}\right], \#(41)
$$

and by substituting (41) in (37), $F_{\gamma_{d}}\left(\gamma_{t h}\right)$ can be stated as 


$$
F_{\gamma_{d}}\left(\gamma_{t h}\right)=\prod_{k=1}^{K}\left[1-e^{-\left(\frac{\gamma_{t h}^{2}}{2 * \Omega_{s r_{n, k}}^{2}}\right)} \prod_{n=1}^{N} e^{-\left(\frac{\gamma_{t h}^{2}}{2 * \Omega_{r_{n, k}}^{2}}\right)}\right], \#(42)
$$

For i.i.d. Rayleigh fading channels the above equation can be further simplified as

$$
F_{\gamma_{d}}\left(\gamma_{t h}\right)=\left[1-e^{-\left(\frac{\gamma_{t h}^{2}}{2 * \Omega_{s r_{n, k}}^{2}}\right)} \prod_{n=1}^{N} e^{-\left(\frac{\gamma_{t h}^{2}}{2 * \Omega_{r_{n, k} d}^{2}}\right)}\right]^{K} . \#(43)
$$

Applying the mathematical identity (34) to (43), $F_{\gamma_{d}}\left(\gamma_{t h}\right)$ can be written as

$$
F_{\gamma_{d}}\left(\gamma_{t h}\right)=\sum_{j=0}^{K}\left(\begin{array}{c}
K \\
j
\end{array}\right)(-1)^{j} e^{-j\left(\frac{\gamma_{t h}^{2}}{2 * \Omega_{s r_{n, k}}^{2}}\right)} \prod_{n=1}^{N} e^{-j\left(\frac{\gamma_{t h}^{2}}{2 * \Omega_{r_{n, k}}^{2}}\right)}
$$

Therefore, the OP for the multiple DF relays with multiple DF hops with BRS over i.i.d. Rayleigh fading channels for cooperative nano relaying can be written as follows by substituting the values for $\Omega_{s r_{n, k}}=\alpha_{s r_{n, k}} * \beta_{s r_{n, k}}$ and $\Omega_{r_{n, k} d}=\alpha_{r_{n, k} d} * \beta_{r_{n, k} d}$ in (44).

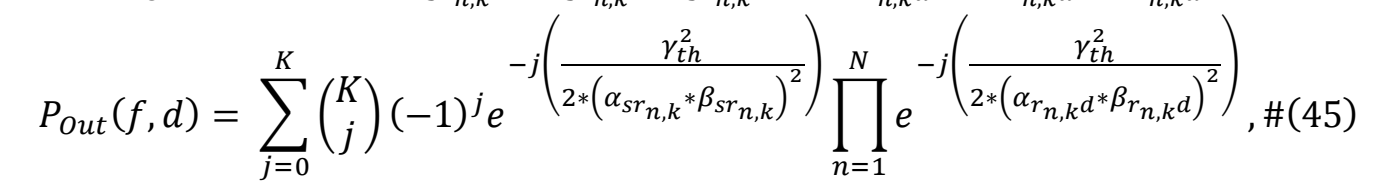

where $\alpha_{s r_{n, k}}=\frac{s_{s r_{n, k}}(f, d) * A_{s r_{n, k}}^{-1}(f, d)}{N_{s r_{n, k}}(f, d)}$ and $\alpha_{r_{n, k} d}=\frac{S_{r_{n, k} d}(f, d) * A_{r_{n, k} d}^{-1}(f, d)}{N_{r_{n, k} d}(f, d)} . S_{i}(f, d)$ is the transmitted signal power, $A_{i}(f, d)$ is the total pathloss and $N_{i}(f, d)$ is the noise power. $\beta_{s r_{n, k}}$ and $\beta_{r_{n, k} d}$ are the Rayleigh fading channels parameters.

\section{Results and Discussion}

The total signal energy has been kept at a constant 800pJ, this number has been taken after considering energy harvesting devices for nano-devices, such as piezoelectric nanogenerators [24], [25], [26], [27]. Monte-Carlo simulations have verified all the analysis. A standard medium of 1 percent water vapor molecules has been considered for all scenarios. Furthermore, for scenarios considered the value of $\beta_{i}=5$. The transmission windows have been chosen after taking into consideration of the following [14], [17], [28], [29]. According to [14], transmission windows with minimum attenuation are located at $300 \mathrm{GHz}, 350 \mathrm{GHz}$, $410 \mathrm{GHz}, 670 \mathrm{GHz}$ and $850 \mathrm{GHz}$.

\subsection{Single DF Relay}

OP analysis for a single DF relay over Rayleigh fading channels for transmission in the windows of $350 \mathrm{GHz}$ band, $410 \mathrm{GHz}$ band and 670GHz band are shown in Fig. 4. From Fig. 4 , it is possible to infer that the best OP performance can be obtained in the $350 \mathrm{GHz}$ band window. 


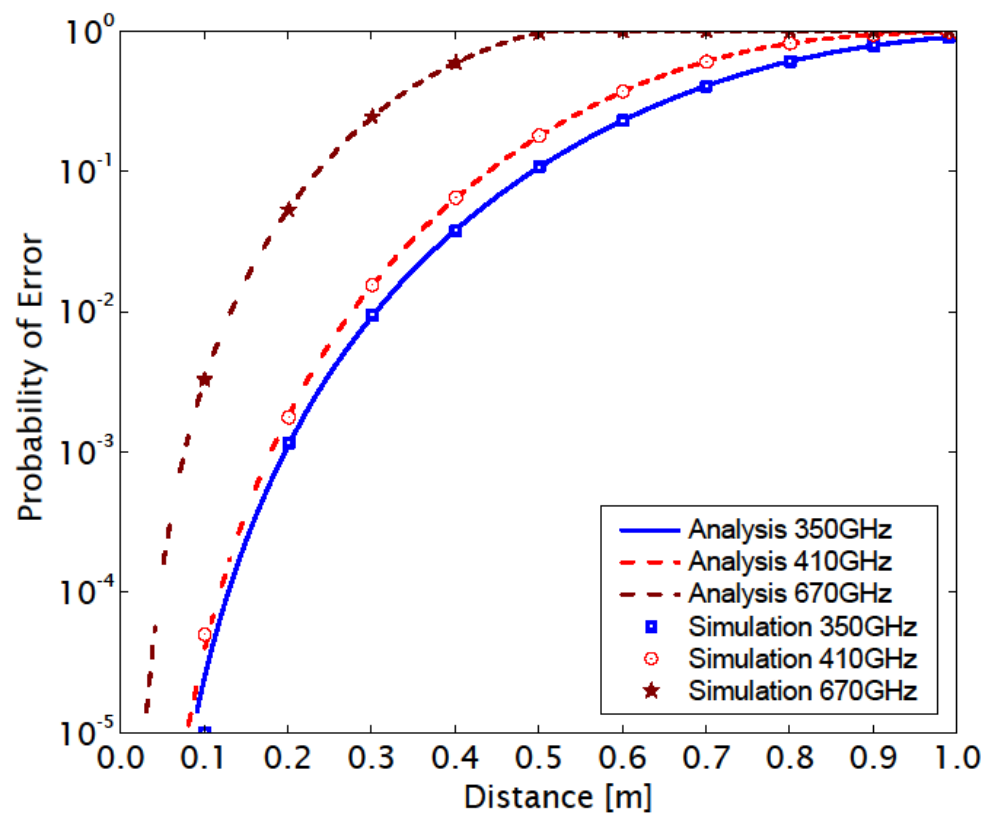

Fig. 4. Outage Probability vs Distance for data transmission over the transmission windows of 350GHz, 410GHz and 670GHz for a Single DF Relay.

\subsection{Multiple DF Relays with BRS}

OP for a multiple DF relays over i.i.d. Rayleigh fading channels with BRS scheme over the transmission window of $350 \mathrm{GHz}$ is shown in Fig. 5. In Fig. 6, for the transmission window of $410 \mathrm{GHz}$, and Fig. 7 for the transmission window of $670 \mathrm{GHz}$. Where, it has been analyzed for the scenarios of 2, 3 and 5 Relays. Furthermore in Fig. 8, the OP performance for transmission windows of $350 \mathrm{GHz}, 410 \mathrm{GHz}$, and $670 \mathrm{GHz}$ are compared.

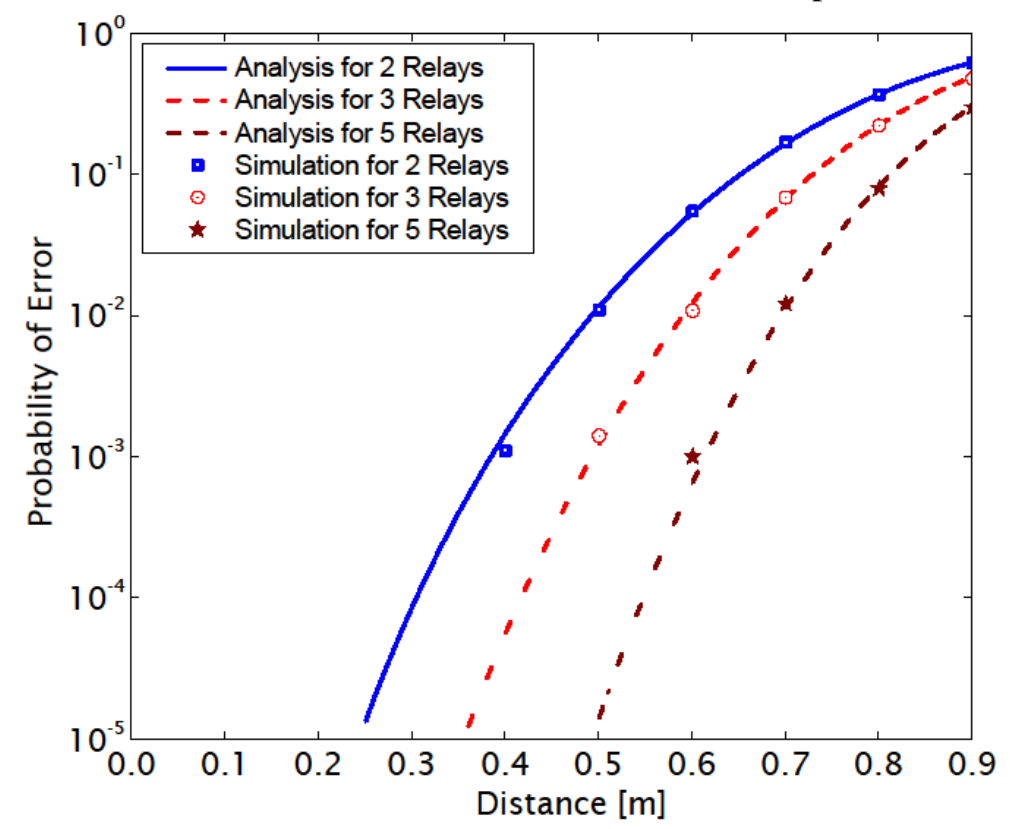

Fig. 5. Outage Probability vs Distance, for data transmission over the transmission window of 350GHz for Mulitple DF Relays with BRS. 


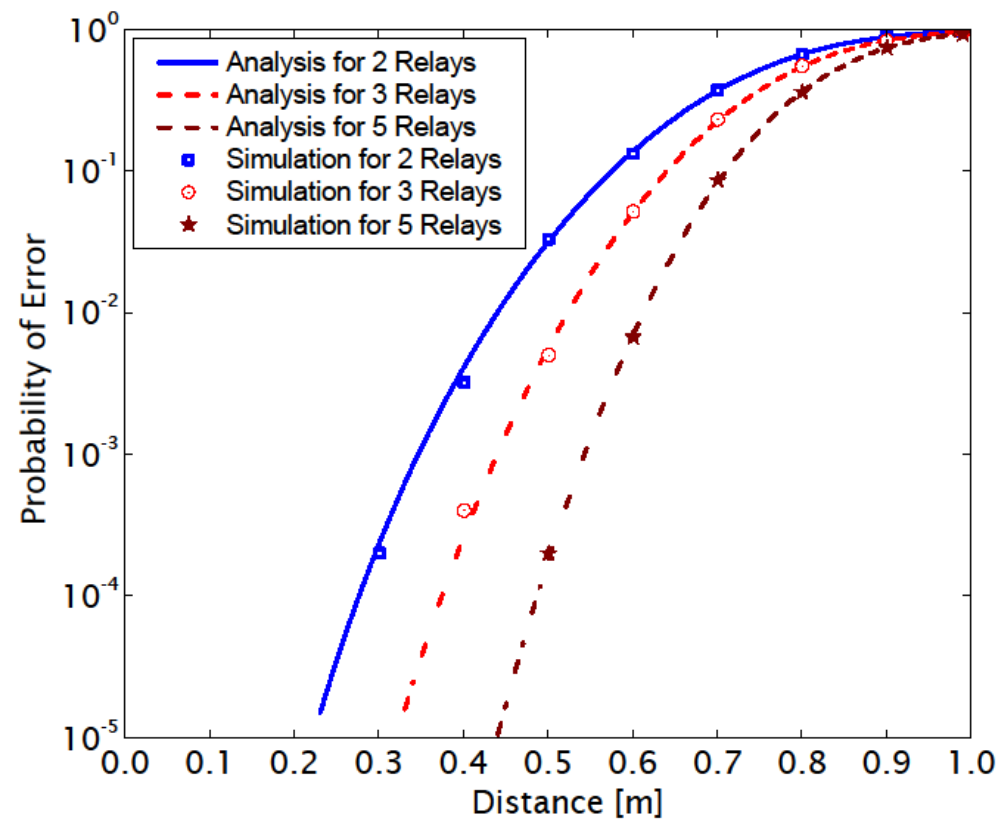

Fig. 6. Outage Probability vs Distance for data transmission over the transmission window of $410 \mathrm{GHz}$ for Multiple DF Relays with BRS.

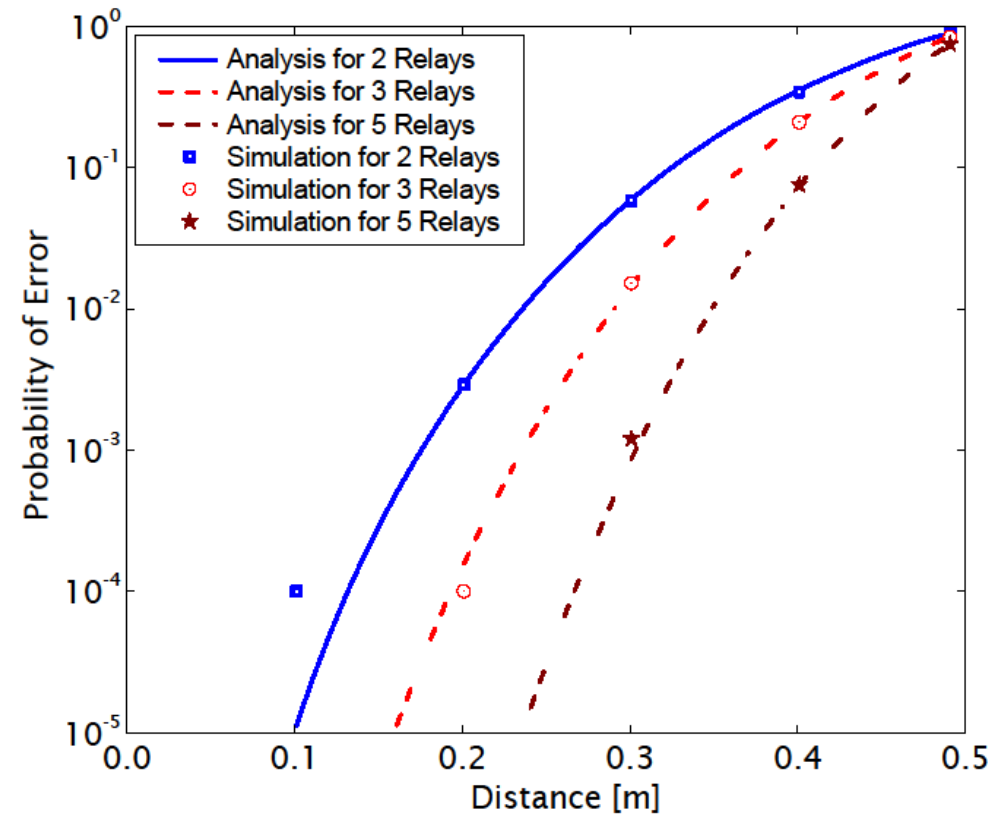

Fig. 7. Outage Probability vs Distance for data transmission over the transmission window of $670 \mathrm{GHz}$ for Multiple DF Relays with BRS. 


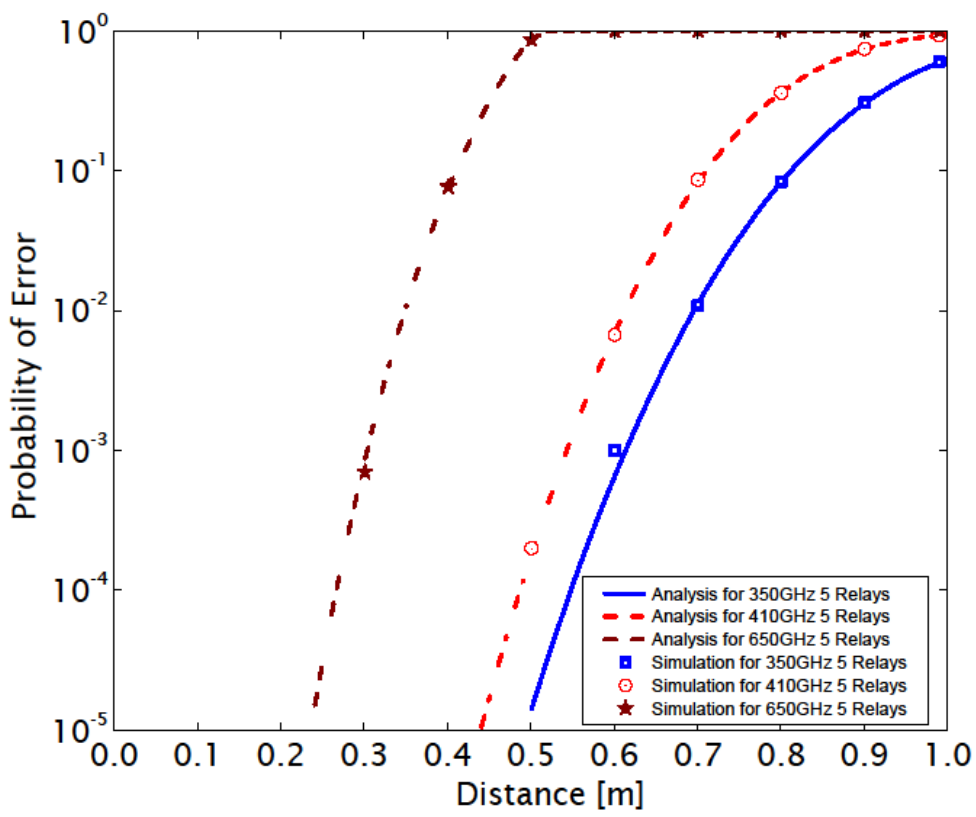

Fig. 8. A comparison of Outage Probability vs Distance for data transmission over the transmission windows of 350GHz, $410 \mathrm{GHz}$ and $670 \mathrm{GHz}$ for Multiple DF Relays with BRS.

It is possible to see from the above mentioned figures that by increasing the number of relays in the network, better OP performance can be obtain. From Fig. 8, it is evident that the best OP performance has been archived in the $350 \mathrm{GHz}$ transmission window.

\subsection{Multiple DF Relays with Multiple DF Hops using BRS}

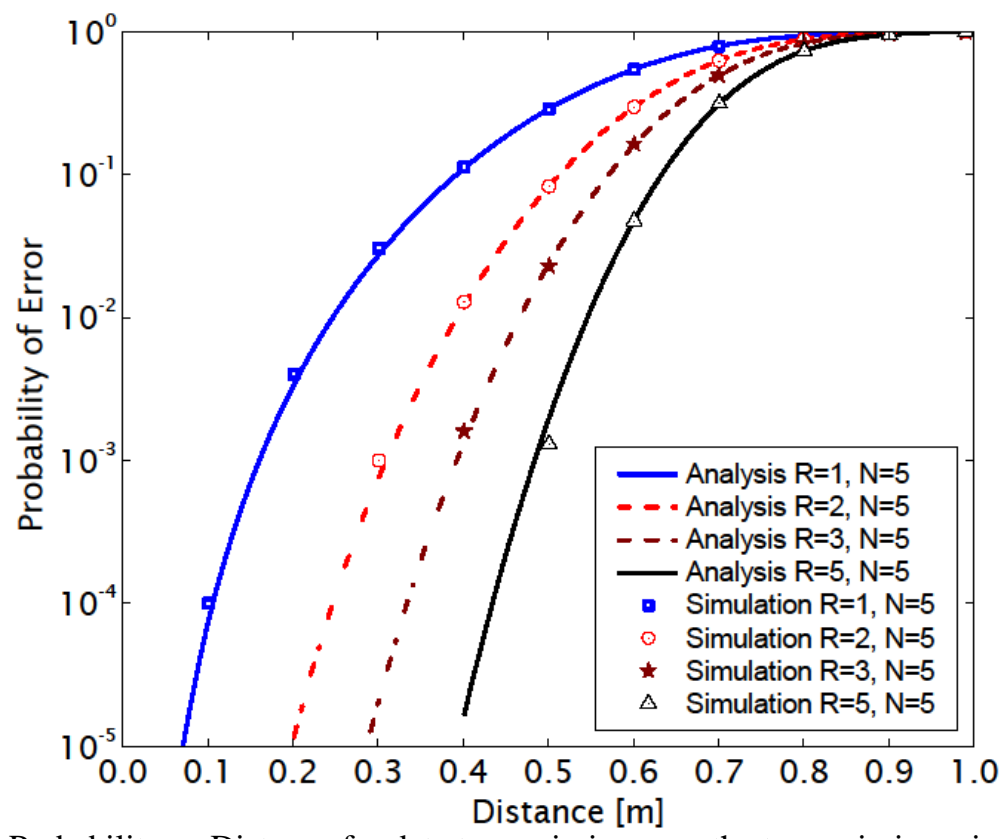

Fig. 9. Outage Probability vs Distance for data transmission over the transmission window of 350GHz for Multiple DF Relays with 5 Hops per Relay. 


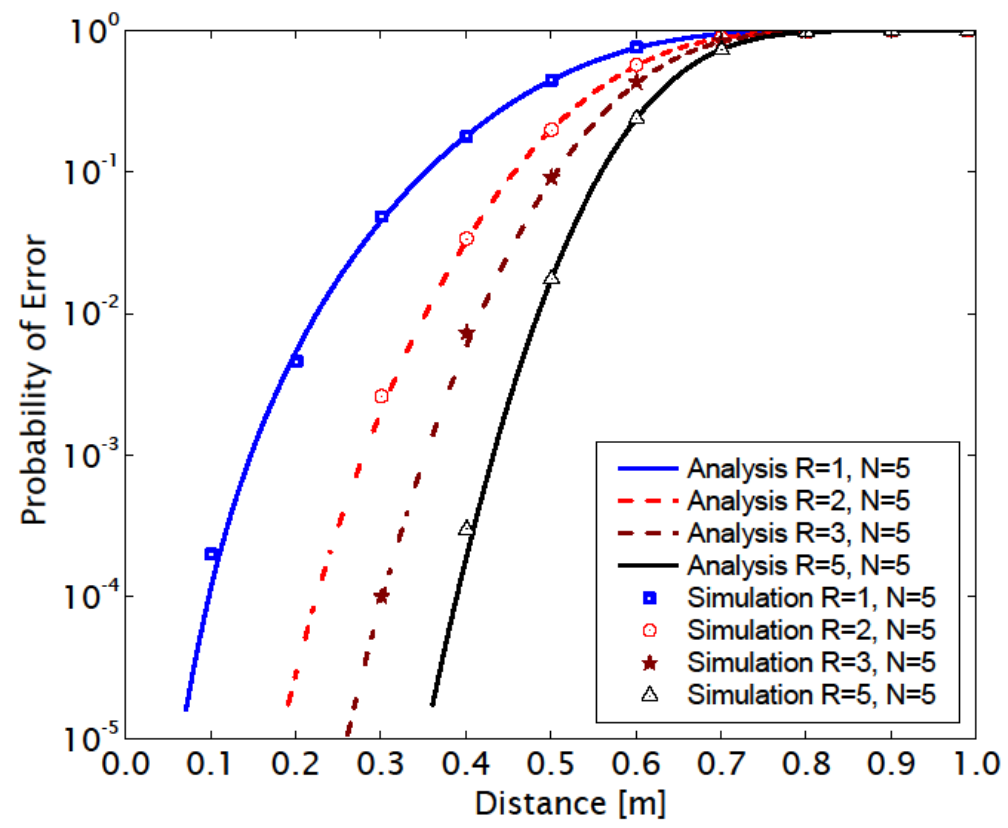

Fig. 10. Outage Probability vs Distance for data transmission over the transmission window of 410GHz for Multiple DF Relays with 5 Hops per Relay.

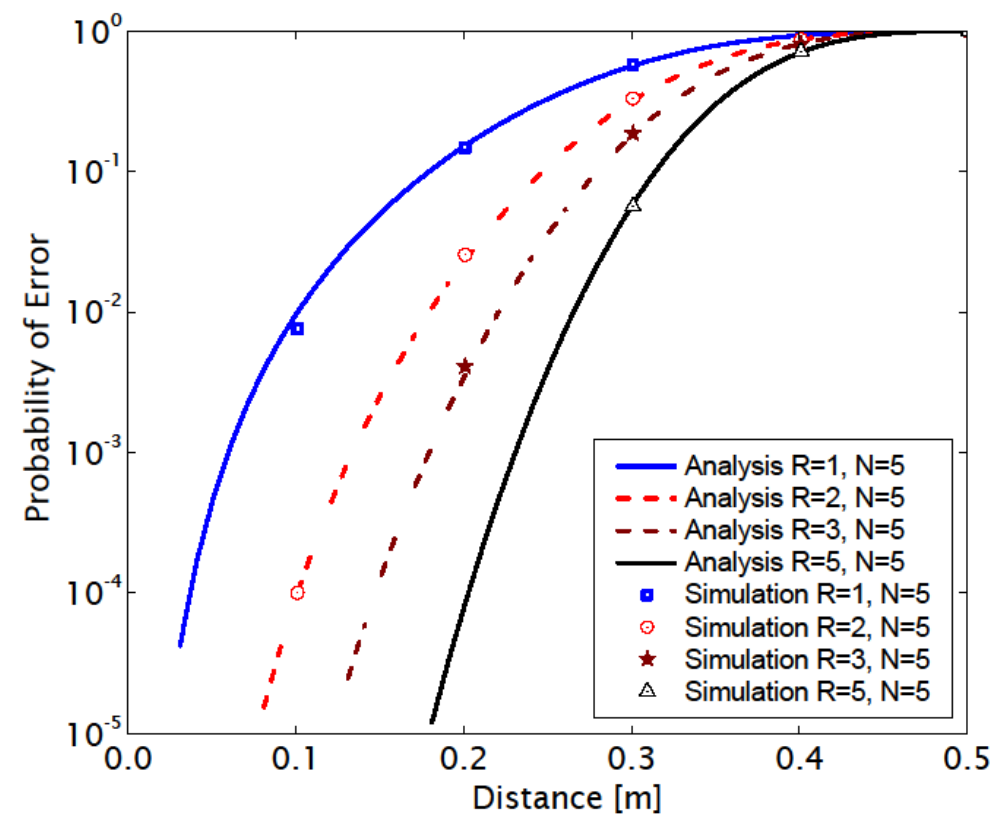

Fig. 11. Outage Probability vs Distance for data transmission over the transmission window of 670GHz for Multiple DF Relays with 5 Hops per Relay. 


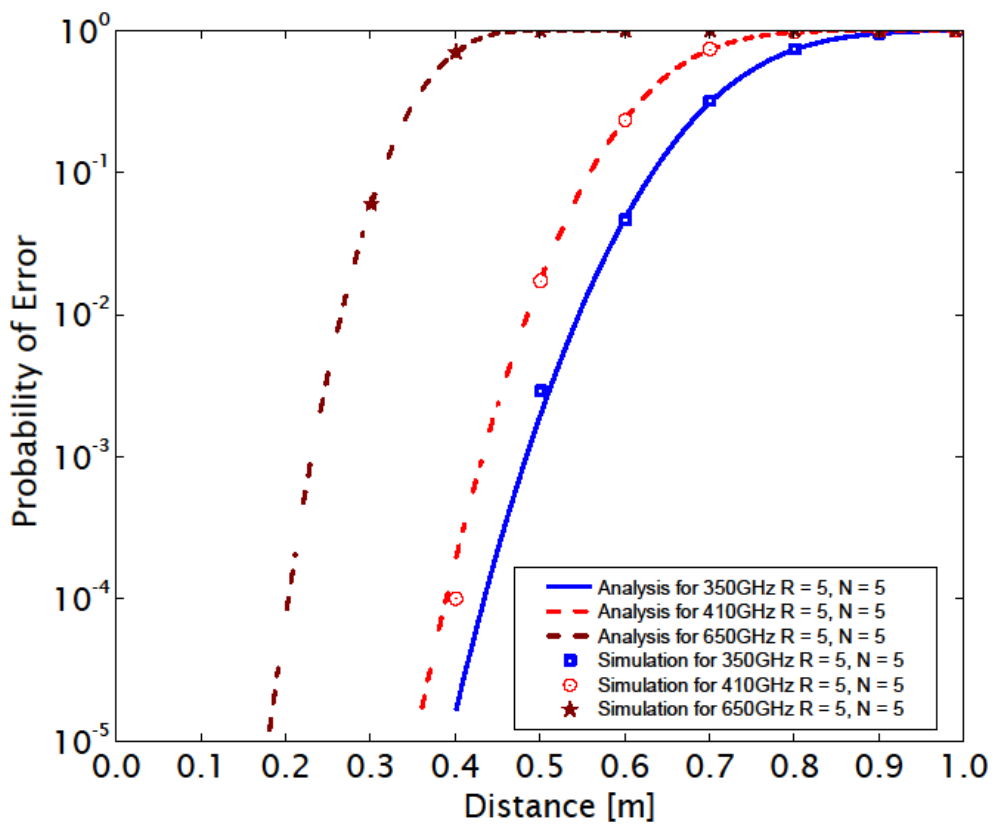

Fig. 12. A comparison of Outage Probability vs Distance for data transmission over the transmission windows of $350 \mathrm{GHz}, 410 \mathrm{GHz}$ and $670 \mathrm{GHz}$ for Multiple DF Relays with 5 Hops per Relay.c

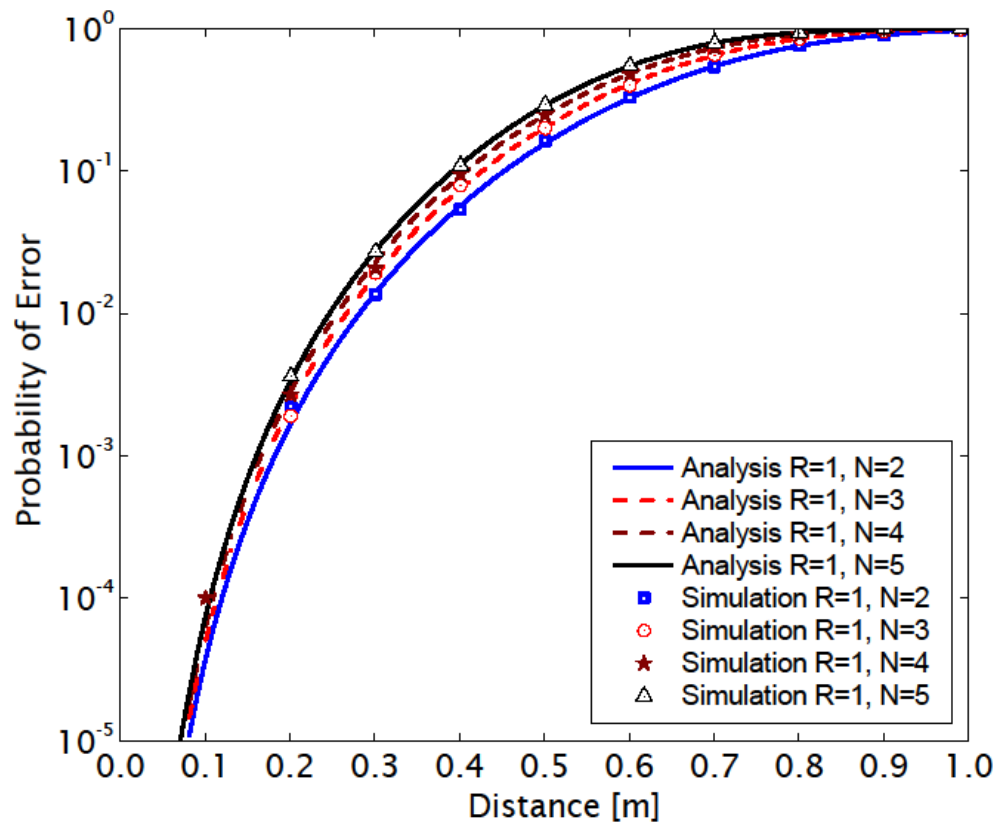

Fig. 13. Outage Probability vs Distance for data transmission over the tranmission window of 350GHz for a Single DF Relay with Multiple Hops. 


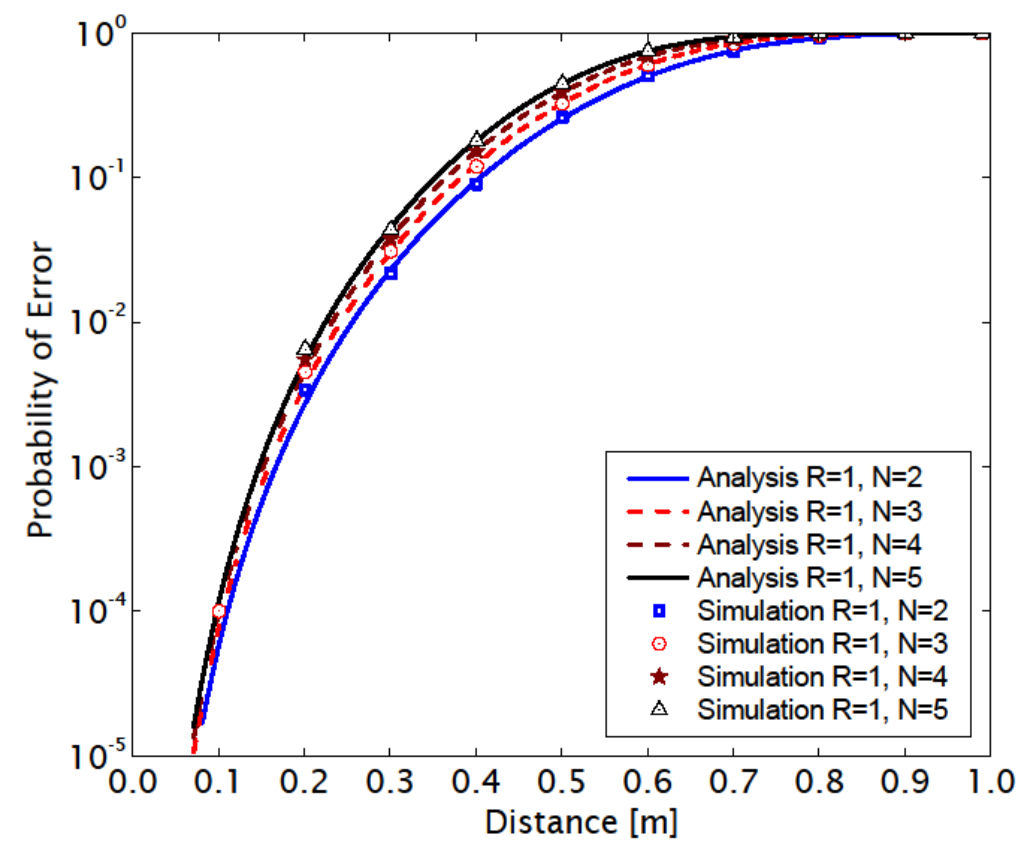

Fig. 14. Outage Probability vs Distance for data transmission over the transmission window of 410GHz for Single DF Relay with Multiple Hops.

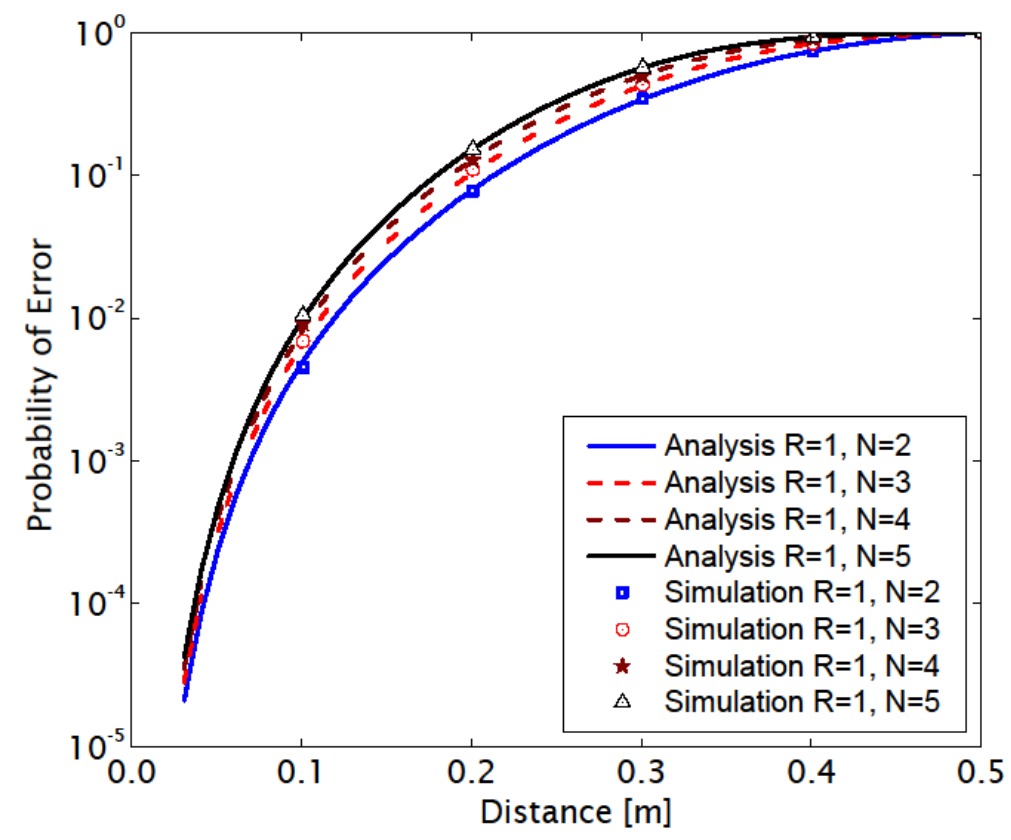

Fig. 15. Outage Probability vs Distance for data transmission over the transmission window of $670 \mathrm{GHz}$ for Single DF Relay with Multiple Hops. 


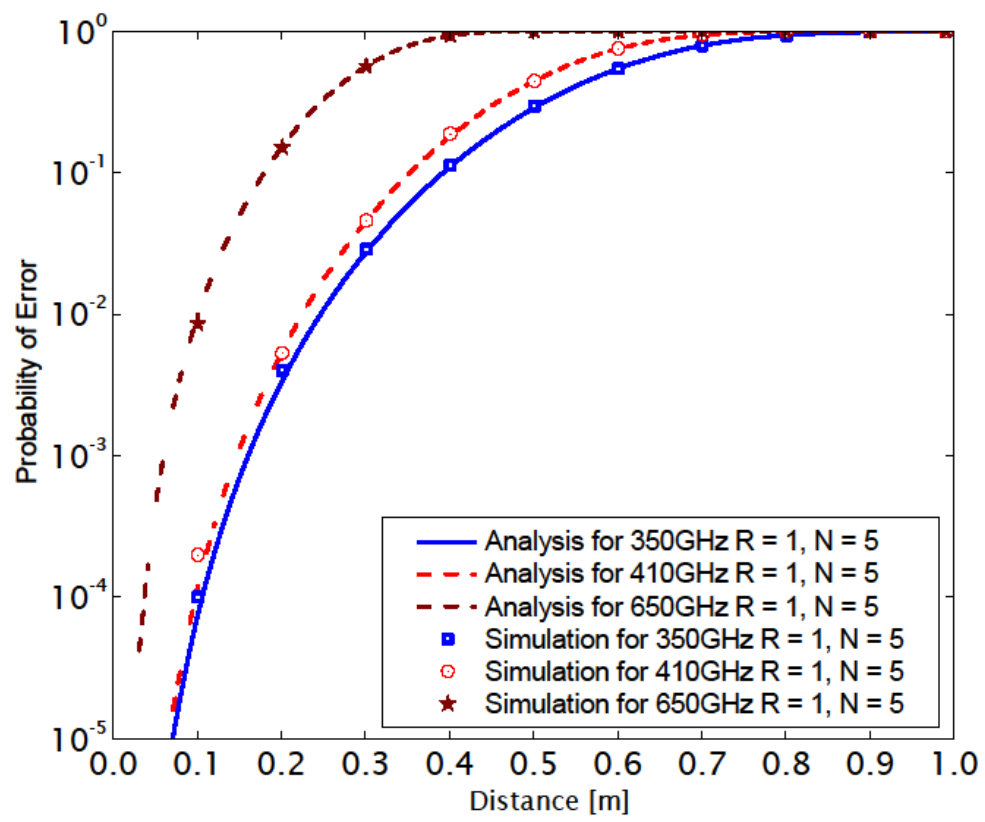

Fig. 16. A comparison of Outage Probability vs Distance for data transmission over the transmission windows of 350GHz, 410GHz and 670GHz for Single DF Relay with Multiple Hops.

OP for multiple DF relays with multiple hops in each relay over i.i.d. Rayleigh fading channels with BRS scheme are shown in Fig. 9 through Fig. 16.

In Fig. 9, Fig. 10, and Fig. 11 multiple DF relays with multiple hops have been investigated. For the scenarios considered in the graphs mentioned, there are 5 hops for each relay. It can be observed from the above graphs that by increasing the number of relays from 1 to 5, the OP performance have increased significantly. In Fig. 12, the OP performance for transmission windows in $350 \mathrm{GHz}, 410 \mathrm{GHz}$ and $670 \mathrm{GHz}$ are compared.

In Fig. 13, Fig. 14, and Fig. 15, a single DF relay with multiple hops have been investigated. It is possible to see from the graphs, that by increasing the number of hops from 2 to 5, the OP performance has only been degraded minutely. In Fig. 16, the OP performance for transmission windows at $350 \mathrm{GHz}, 410 \mathrm{GHz}$ and $670 \mathrm{GHz}$ are compared.

\section{Conclusion}

One of the major hurdles of nano communications is the limited amount of transmission power available for nano devices. In this paper, it is shown that by using cooperative communication techniques it is possible to extend the transmission distances through relaying. Terahertz band is capable of very high capacity and very high transmission bit rates. Therefore, cooperative communication among nano devices in the Terahertz band can provide significate improvements to the capabilities of nano-devices.

The results have shown that by increasing the number of relays it is possible to get better OP performance according to Fig. 5 through Fig. 7 and Fig. 9 through Fig. 11. Furthermore, from Fig. 13 through Fig. 15, it is evident that by increasing the number of hops per relay from 2 to 5 the OP performance was not affected significantly. Therefore, the range between source nano machine and destination nano machine can be significantly boosted through cooperative communication. Which will enable nano technology in diverse fields ranging from national security application to health care monitoring systems. From Fig. 4, Fig. 8 and 
Fig. 12 it is possible to see that the best OP performance has been obtained in the $350 \mathrm{GHz}$ transmission window.

Molecular absorption is dependent on frequency and distance. From the Fig. 4, Fig. 8 and Fig. 12 it is possible to see that when the transmission frequency is increased the attenuation due to molecular absorption increases and the transmission distance decreases. Therefore, the relaying distances are dependent on the attenuation due to molecular absorption which is dependent on the frequency of operation and transmission energy. The amount of energy piezoelectric nano-generators are able to produce constantly is around 800pJ [24], [25], [26], [27] at the moment. As the energy generation capability of peiozoelectric nano-generators increases the transmission distances can be further increased and better performance can be obtained.

The analytical results have been verified by Monte-Carlo simulations for all the scenarios in the paper.

\section{References}

[1] I. F. Akyildiz, F. Brunetti and C. BlÃ $\tilde{i z q u e z}$ "Nanonetworks: A new communication paradigm," Computer Networks, vol. 52, no. 12, pp. 2260 - 2279, Aug 2008. Article (CrossRef Link).

[2] I. F. Akyildiz and J. M. Jornet, "Electromagnetic wireless nanosensor networks," Nano Communication Networks, vol. 1, no. 1, pp. 3 - 19, March May 2010. Article (CrossRef Link).

[3] J. M. Jornet and I. F. Akyildiz, "Channel Capacity of Electromagnetic Nanonetworks in the Terahertz Band," in Proc. of Communications (ICC), 2010 IEEE International Conference on, Cape Town, 2010. Article (CrossRef Link).

[4] J. Q. Liu and T. Nakano, "Principles and Methods for Nanomechatronics: Signaling, Structure, and Functions Toward Nanorobots," IEEE Transactions on Systems, Man, and Cybernetics, Part C (Applications and Reviews), vol. 42, no. 3, pp. 357 - 366, May 2012. Article (CrossRef Link).

[5] Y. Okaie, T. Nakano, T. Hara and S. Nishio, "Distributing Nanomachines for Minimizing Mean Residence Time of Molecular Signals in Bionanosensor Networks," IEEE Sensors Journal, vol. 14, no. 1, pp. 218 - 227, Jan 2014. Article (CrossRef Link).

[6] F. Mesiti and I. Balasingham, "Nanomachine-to-Neuron Communication Interfaces for Neuronal Stimulation at Nanoscale," IEEE Journal on Selected Areas in Communications, vol. 31, no. 12, pp. 695 - 704, Dec 2013. Article (CrossRef Link).

[7] J. M. Jornet and I. F. Akyildiz, "Channel Modeling and Capacity Analysis for Electromagnetic Wireless Nanonetworks in the Terahertz Band," IEEE Transactions on Wireless Communications, vol. 10, no. 10, pp. 3211 - 3221, Oct 2011. Article (CrossRef Link).

[8] compiled by the class for Physics of the Royal Swedish Academy of Sciences, "GRAPHENE, Scientific Background on the Nobel Prize in Physics 2010," Physics of the Royal Swedish Academy of Sciences, Stockholm, Oct 2010. Article (CrossRef Link).

[9] P. Avouris, Z. Chen and V. Perebeiros, "Carbon-based electronics," Nature Nanotechnology, vol. 2, no. 10, pp. 605 - 615, Sep 2007. Article (CrossRef Link).

[10] M. Dragoman, A. A. Muller, D. Dragoman, F. Coccetti and R. Plana, "Terahertz antenna based on graphene," Journal of Applied Physics, vol. 107, no. 10, May 2010. Article (CrossRef Link).

[11] J. M. Jornet and I. F. Akyildiz, "Graphene-based nano-antennas for electromagnetic nanocommunications in the terahertz band," in Proc. of the Fourth European Conference on Antennas and Propagation, Barcelona, 2010. Article (CrossRef Link). 
[12] I. Llatser, C. Kremers, A. Cabellos-Aparicio, J. M. Jornet, E. Alarc $\tilde{A}^{3} n$ and D. N. Chigrin, "Graphene-based nano-patch antenna for terahertz radiation," Photonics and Nanostructures Fundamentals and Applications, vol. 10, no. 4, pp. 353 - 358, May 2012. Article (CrossRef Link).

[13] J. C. Wiltse, "History of Millimeter and Submillimeter Waves," IEEE Transactions on Microwave Theory and Techniques, vol. 32, no. 9, pp. 1118 - 1127, Sep 1984. Article (CrossRef Link).

[14] T. Kleine-Ostmann and T. Nagatsuma, "A Review on Terahertz Communications Research," Journal of Infrared, Millimeter, and Terahertz Waves, vol. 32, no. 2, pp. 143 - 171, Feb 2011. Article (CrossRef Link).

[15] J. Federici and L. Moeller, "Review of terahertz and subterahertz wireless communications," Journal of Applied Physics, vol. 107, no. 11, Jun 2010. Article (CrossRef Link).

[16] H. J. Song and T. Nagatsuma, "Present and Future of Terahertz Communications," IEEE Transactions on Terahertz Science and Technology, vol. 1, no. 1, pp. 256 - 263, Sep 2011. Article (CrossRef Link).

[17] R. Piesiewicz, T. Kleine-Ostmann, N. Krumbholz, D. Mittleman, M. Koch, J. Schoebei and T. Kurner, "Short-Range Ultra-Broadband Terahertz Communications: Concepts and Perspectives," IEEE Antennas and Propagation Magazine, vol. 49, no. 6, pp. 24 - 39, Dec 2007. Article (CrossRef Link).

[18] H. Yu, B. Ng and W. K. Seah, "Forwarding Schemes for EM-based Wireles Nanosensor Networks in the Terahertz Band.," in Proc. of Second Annual International Conference on Nanoscale Computing and Communication, New York, NY, 2015. Article (CrossRef Link).

[19] F. Afsana, S. A. Mamun, M. S. Kaiser and M. R. Ahmed, "Outage Capacity Analysis of Cluster-based Forwarding Scheme for Body Area Network using Nano-electromagnetic Communication," in Proc. of 2nd International Conference on Electrical Information and Communication Technology, 2015. Article (CrossRef Link).

[20] J. H. Van Vleck and V. F. Weisskopf, "On the Shape of Collision-Broadened Lines," Rev. Mod. Phys., vol. 17, no. 2 - 3, pp. 227 - 236, Apr 1945. Article (CrossRef Link).

[21] S. A. Clough, F. X. Kneizys and R. W. Davies, "Line shape and the water vapor continuum," Atmospheric Research, vol. 23, no. 3, pp. 229 - 241, Jun 1989. Article (CrossRef Link).

[22] V. N. Q. Bao, T. Doung, D. B. da Costa, G. Alexandropoulos and A. Nallanathan, "Cognitive amplify-and-forward relaying with best relay selection in non-identical rayleigh fading," IEEE Commun. Lett., vol. 17, no. 3, pp. 475-478, 2013. Article (CrossRef Link).

[23] T. Doung, V. Bao, G. Alexandropoulos and H.-J. Zepernick, "Cooperative spectrum sharing networks with AF relay and selection diversity," Electronic Letter, vol. 47, no. 20, pp. 11491151, 2011. Article (CrossRef Link).

[24] J. M. Jornet and I. F. Akyildiz, "Joint Energy Harvesting and Communication Analysis for Perpetual Wireless Nanosensor Networks in the Terahertz Band," IEEE Transactions on Nanotechnology, vol. 11, no. 3, pp. 570 - 580, May 2012. Article (CrossRef Link).

[25] Z. L. Wang, "Towards Self-Powered Nanosystems: From Nanogenerators to Nanopiezotronics," Advanced Functional Materials, vol. 18, no. 22, pp. 3553 - 3567, Nov 2008. Article (CrossRef Link).

[26] S. Xu, B. J. Hansen and Z. L. Wang, "Piezoelectric-nanowire-enabled power source for driving wireless microelectronics," Nature Communication, vol. 1, no. 7, pp. 1 - 5, Oct 2010. Article (CrossRef Link).

[27] S. Xu, Y. Qin, C. Xu, Y. Wei, R. Yang and Z. L. Wang, "Self-powered nanowire devices," Nature nanotechnology, vol. 5, no. 5, pp. 366 - 373, May 2010. Article (CrossRef Link). 
[28] P. Boronin, V. Petrov, D. Moltchanov, Y. Koucheryavu and J. M. Jornet, "Capacity and throughput analysis of nanoscale machine communication through transparency windows in the terahertz band," Nano Communication Networks, vol. 5, no. 3, pp. 72-82, 2014. Article (CrossRef Link).

[29] R. Piesiewicz, J. Jemai, M. Koch and T. Kurner, "THz channel characterization for future wireless gigabit indoor communication systems," in Proc. of SPIE, vol. 5727, pp. 166-176, 2005. Article (CrossRef Link).

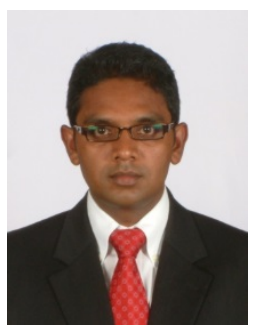

A. Chaminda J. Samarasekera Received the B.Sc degree in Avionics Engineering Technology from Embry-Riddle Aeronautical Engineering, Daytona Beach, Florida, USA, in 2004 and M.Sc. degree in Electrical Engineering in the area of Radio Communication from Blekinge Institute of Technology, Karlskrona, Sweden. Currently working towards a Ph.D. degree in Electronics and Radio Engineering at Kyung Hee University, Yongin-si, Korea. His current research interest include wireless communication, Terahertz communication, Nano Communications, and Cooperative Communications.

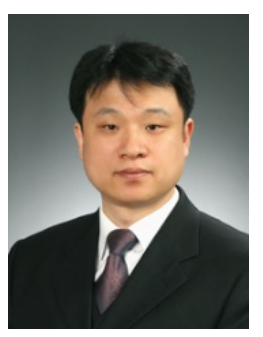

Hyundong Shin (S'01-M'04-SM'11) received the B.S. degree in electronics engineering from Kyung Hee University (KHU), Yongin-si, Korea, in 1999 and M.S. and Ph.D. degrees in electrical engineering from Seoul National University, Seoul, Korea, in 2001 and 2004, respectively. During his postdoctoral research at the Massachusetts Institute of Technology (MIT) from 2004 to 2006, he was with the Wireless Communication and Network Sciences Laboratory within the Laboratory for Information Decision Systems (LIDS). In 2006, Dr. Shin joined the KHU, where he is now an Associate Professor at the Department of Electronics and Radio Engineering. His research interests include wireless communications and information theory with current emphasis on MIMO communication systems, cooperative communication networks, cognitive radio and networks, network interference, vehicular communication networks, physical-layer security, location-aware radios and networks, molecular communications, and quantum information science. Dr. Shin was honored with the Knowledge Creation Award in the field of Computer Science from Korean Ministry of Education, Science and Technology (2010). He received the IEEE Communications Society's Guglielmo Marconi Prize Paper Award (2008) and William R. Bennett Prize Paper Award (2012). He served as a Technical Program Co-chair for the IEEE WCNC (PHY Track 2009) and the IEEE Globecom (Communication Theory Symposium 2012, Cognitive Radio and Networks Symposium 2016). He was an Editor for IEEE Transactions on Wireless Communications (2007-2012) and IEEE Communications Letters (2013-2015). 